This is an Open Access article distributed under the terms of the Creative Commons Attribution-Noncommercial License, which permits unrestricted use, distribution, and reproduction in any noncommercial medium, provided the original work is properly cited.

\title{
DEPLETION STUDY OF SI AND FE WITH MID- AND FAR-INFRARED IMAGING SPECTROSCOPY WITH SPICA
}

\author{
Y. Okada ${ }^{1}$, T. Onaka ${ }^{2}$, H. Kaneda ${ }^{3}$, and I. Sakon ${ }^{2}$ \\ ${ }^{1}$ I. Physikalisches Institut, Universität zu Köln, Zülpicher Str. 77, 50937 Köln, Germany \\ ${ }^{2}$ Department of Astronomy, Graduate School of Science, University of Tokyo, 7-3-1 Hongo, Bunkyo-ku, Tokyo 113-0033, Japan \\ ${ }^{3}$ Graduate School of Science, Nagoya University, Furo-cho, Chikusa-ku, Nagoya 464-8602, Japan
}

\section{Abstract}

Ionic emission lines, such as [Si II] $35 \mu \mathrm{m}$, [Fe II] $26 \mu \mathrm{m}$, and [Fe III] $23 \mu \mathrm{m}$, can be used to investigate the gas-phase abundance of $\mathrm{Si}$ and $\mathrm{Fe}$, which are key elements of the constituents of interstellar dust grains. The depletion study of $\mathrm{Si}$ and Fe provides crucial information on the chemical composition of dust. The investigation of the spatial distribution and the correlation with the physical properties of the environment in active regions is important for the understanding of the evolution of the interstellar matter. Previous studies showed that the gas-phase Si abundance is much larger than that in cool interstellar clouds and much less depleted than Fe. However, the depletion of these metals in active regions still has large uncertainties due to the varying physical properties in the large aperture as well as the insufficient sensitivity. We explore the strategy to estimate the physical properties using midand far-infrared emission lines that SPICA can observe.

Key words: Infrared:ISM - ISM:abundances - lines and bands - H II regions - Missions: SPICA

\section{INTRODUCTION}

The interstellar matter (ISM) consists of gas and dust, and their life-cycle is a big issue to be examined in astronomy. One approach to probe the evolution of the ISM is depletion studies. The depletion is defined as the lack of atoms in the gas-phase compared to the total abundance of a certain element. This provides key information on the chemical composition of dust grains in various environments.

Among several methods to investigate the chemical composition of dust grains, the depletion study in infrared wavelength has the following advantages: (1) the interpretation of the emission lines is rather straightforward than that of spectral feature from dust grains, (2) the midinfrared (MIR) and far-infrared (FIR) emission lines are less sensitive to the extinction and the electron temperature in H II regions, (3) key elements of dust grains (i.e. Si and $\mathrm{Fe}$ ) have ionic emission lines in MIR, and (4) the diagnostic emission lines for physical properties of the ionized and the photodissociation region (PDR) can be observed at the same time.

\section{Previous studies and its Limitations}

Previous studies show that over $10 \%$ of $\mathrm{Si}$ atoms are in the gas-phase in star-forming regions (Okada et al. 2008, 2006, 2003; Mizutani et al. 2004; Fuente et al. 2000; Colgan et al. 1993), whereas the gas-phase abundance of Si in the cool ISM is $\sim 5 \%$ (Savage \& Sembach 1996). On the other hand, the gas-phase Fe abundance is below $1 \%$ or a few \% of the solar abundance (Okada et al. 2008; Lebouteiller et al. 2008; van den Ancker et al. 2000; Timmermann et al. 1996). Okada et al. (2008) show that the Si abundance ranges from $10 \%$ to $100 \%$ of the solar abundance, whereas that of $\mathrm{Fe}$ is from $1 \%$ to $10 \%$ of the solar abundance from the observations with the Infrared Spectrograph (IRS; Houck et al. 2004) onboard Spitzer (Werner et al. 2004) in 14 star-forming regions (figure 1). They suggested that a larger fraction of $\mathrm{Si}$ atoms are included in dust grains which are destroyed easily in H II regions, probably by the UV radiation; mantles, organometallic complexes, or small grains.

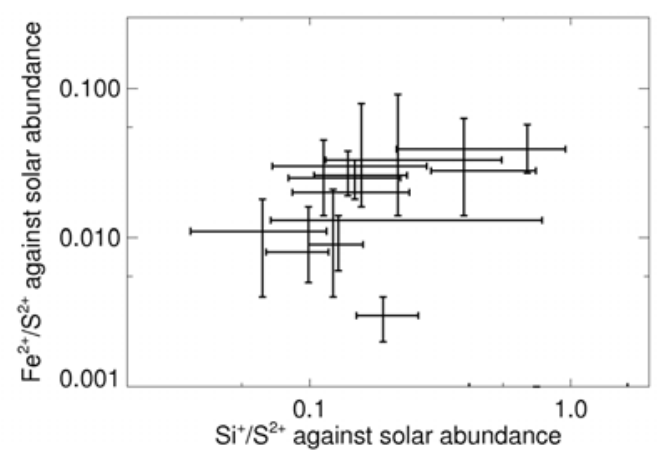

Figure 1. $\mathrm{Fe}^{2+} / \mathrm{S}^{2+}$ against $\mathrm{Si}^{+} / \mathrm{S}^{2+}$ normalized by the solar abundance in 14 star-forming regions (Okada et al. 2008).

Though it is certain that the IRS will provide much more samples in near future, there are some limitations with the present observational data. First, the origin of [Si II] $35 \mu \mathrm{m}$ and [Fe II] $26 \mu \mathrm{m}$ is uncertain in most cases. Since the first ionization potentials of $\mathrm{Si}$ and Fe are less than $13.6 \mathrm{eV}$, these lines can originate both from the ionized gas and PDR gas. Mizutani et al. (2004) took the correlation between [Si II] $35 \mu \mathrm{m}$ and [N II] $122 \mu \mathrm{m}$, which 
traces the ionized gas, and [O I] $63 \mu \mathrm{m}$, which traces the PDR gas, and express the [Si II] $35 \mu \mathrm{m}$ intensity with the linear combination of [N II] $122 \mu \mathrm{m}$ and [O I] $63 \mu \mathrm{m}$ to distinguish the contribution from the ionized and PDR gas in the Carina region. This strategy needs a sufficient observed area and thus datasets for it are very limited at present.

Another problem is the lack of information on physical properties, such as the electron density in $\mathrm{H}$ II regions and the gas density and the radiation field strength in PDRs, in similar aperture size with those of the emission lines from $\mathrm{Si}$ and $\mathrm{Fe}$ ions. The diffuse lowly ionized gas is traced by [N II] lines in FIR wavelength range (see Table 1), and the PDR properties can be derived by FIR line and continuum emissions. However, the Long-Wavelength Spectrometer (LWS; Clegg et al. 1996) onboard the Infrared Space Observatory (ISO; Kessler et al. 1996) has a beam size of $87^{\prime \prime}$ at $63 \mu \mathrm{m}$ (Gry et al. 2003), whereas the slit size of the IRS Long-High (LH) module, which covers [Si II] $35 \mu \mathrm{m}$ and [FeII] $26 \mu \mathrm{m}$, is $11.1^{\prime \prime} \times 22.3^{\prime \prime}$. Okada et al. (2008) observed 4 positions with the IRS within the aperture of the LWS to take account of the difference in the aperture size, but they also found large variations in line intensities within these 4 positions.

\section{Strategy with SPICA}

We propose to make imaging spectroscopic observations both in the MIR and FIR for extensive studies of the depletion of $\mathrm{Si}$ and $\mathrm{Fe}$ in the ISM. The obtained maps of various line ratios will provide the spatial distributions of the physical properties and abundance ratios. Together with key lines of [Si II] $35 \mu \mathrm{m}$, [Fe II] $26 \mu \mathrm{m}$, and [Fe III] $23 \mu \mathrm{m}$, [Si II] $33 \mu \mathrm{m},[\mathrm{N} \mathrm{II}] 122 \mu \mathrm{m},[\mathrm{O}$ I] $146 \mu \mathrm{m}$, and Hu $\alpha$ $12.4 \mu \mathrm{m}$ lines will be used as reference lines to derive the gas-phase abundance of $\mathrm{Si}$ and Fe. In addition, several line ratios will be used to derive the physical properties: [N II] $122 \mu \mathrm{m} / 205 \mu \mathrm{m}$ and [Si II] $18.7 \mu \mathrm{m} / 33 \mu \mathrm{m}$ for the electron density, [O I] $146 \mu \mathrm{m} / \mathrm{FIR}$ for the PDR properties, and so on. In the following, we show the detailed strategy. We summarize the properties of fine-structure lines in the ionized gas that are expected to be detected with SPICA in Table 1.

\subsection{The ORIGIN OF [SIII] $35 \mu \mathrm{M}$ AND [FE II] $26 \mu \mathrm{M}$}

To estimate the contribution of the ionized and PDR gas to the [Si II] $35 \mu \mathrm{m}$ and [Fe II] $26 \mu \mathrm{m}$ emissions, we take correlations of these line intensities with the [N II] $122 \mu \mathrm{m}$ and [O I] $146 \mu \mathrm{m}$. We use [O I] $146 \mu \mathrm{m}$ as a tracer of the PDR gas rather than [O I] $63 \mu \mathrm{m}$, since the former is optically thin. Mapping observations of a sufficient area are crucial for comparing the data observed with different instrument (MIR and FIR) and deriving a reliable coefficient of the relation.
There is another possibility for [Fe II] $26 \mu \mathrm{m}$. The [Fe II] $18 \mu \mathrm{m}$ emission comes from the transition with the upper level of $\sim 3500 \mathrm{~K}$ (figure 2), and thus it originates only from the ionized gas. Once the electron density is estimated the [Fe II] $26 \mu \mathrm{m} / 18 \mu \mathrm{m}$ in the ionized gas can be predicted, and thus we can distinguish the contribution to the observed [FeII] $26 \mu \mathrm{m}$ intensity from the ionized gas and the PDR gas. For this strategy, a high sensitivity to detect [Fe II] $18 \mu \mathrm{m}$ and the resolution of $R>500$ to distinguish the [Fe II] line in $17.94 \mu \mathrm{m}$ from [P III] $17.885 \mu \mathrm{m}$ are needed.

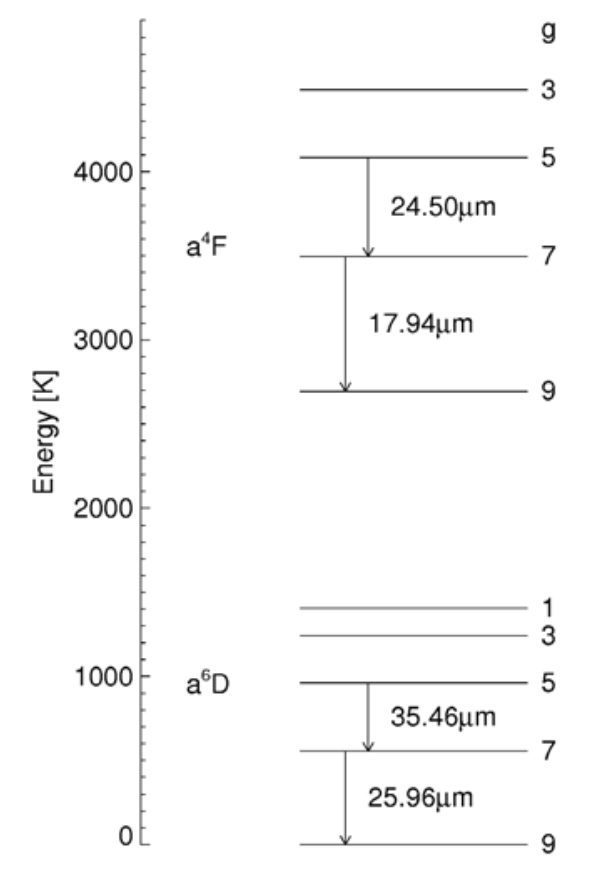

Figure 2. The energy level of $\mathrm{Fe}^{+}$below $5000 \mathrm{~K}$.

\subsection{The Physical PROPERTIES OF ENVIRONMENTS}

The estimate of the physical properties is essential to derive the abundance ratios from the line intensity ratios. In the ionized region, the electron density should be derived. The ratio of MIR and FIR forbidden emission lines from the same ion is a good tracer of the electron density and is insensitive to the electron temperature (figure 3). As figure $3 \mathrm{a}$ and $\mathrm{b}$ show, [S III] $18.7 \mu \mathrm{m} / 33 \mu \mathrm{m}$ is sensitive to the electron density for the range of $10^{4}-10^{5} \mathrm{~cm}^{-3}$, and [N II] $122 \mu \mathrm{m} / 205 \mu \mathrm{m}$ does for $10^{2}-10^{3} \mathrm{~cm}^{-3}$. Thus both ratios should be used to trace a wide range of the electron density.

The PDR properties such as the gas density $(n)$ and the strength of the radiation field $\left(G_{0}\right)$ are derived using the PDR models. As shown in Okada et al. (2003), the 
Table 1. Fine-Structure lines in the ionized gas that are expected to be detected with SPICA.

\begin{tabular}{|c|c|c|c|c|c|}
\hline Species & $\begin{array}{c}\text { Ionization } \\
\text { potential } \\
(\mathrm{eV})\end{array}$ & Transition & $\begin{array}{c}\text { Wavelength } \\
(\mu \mathrm{m})\end{array}$ & $\begin{array}{c}\text { Energy level of } \\
\text { upper state } \\
(\mathrm{K})\end{array}$ & $\begin{array}{c}\text { Critical } \\
\text { density } \\
\left(\mathrm{cm}^{-3}\right)\end{array}$ \\
\hline \multirow[t]{4}{*}[\mathrm{Fe}\mathrm{II}]{} & \multirow[t]{4}{*}{7.87} & ${ }^{6} \mathrm{D}_{7 / 2^{-}}{ }^{6} \mathrm{D}_{9 / 2}$ & 25.99 & 554 & $1.1 \times 10^{4 \mathrm{~b}}$ \\
\hline & & ${ }^{6} \mathrm{D}_{5 / 2^{-}}{ }^{6} \mathrm{D}_{7 / 2}$ & 35.46 & 960 & $6.5 \times 10^{3 \mathrm{~b}}$ \\
\hline & & ${ }^{4} \mathrm{~F}_{7 / 2^{-}}{ }^{4} \mathrm{~F}_{9 / 2}$ & 17.94 & 3496 & $2.4 \times 10^{4 \mathrm{~b}}$ \\
\hline & & ${ }^{4} \mathrm{~F}_{5 / 2}-{ }^{4} \mathrm{~F}_{7 / 2}$ & 24.50 & 4083 & $1.4 \times 10^{4 \mathrm{~b}}$ \\
\hline [Si II] & 8.15 & ${ }^{2} \mathrm{P}_{3 / 2^{-}}{ }^{2} \mathrm{P}_{1 / 2}$ & 34.81 & 413 & $1.8 \times 10^{3}$ \\
\hline [C II] & 11.26 & ${ }^{2} \mathrm{P}_{3 / 2^{-}}{ }^{2} \mathrm{P}_{1 / 2}$ & 157.74 & 91.2 & 18 \\
\hline \multirow[t]{2}{*}[\mathrm{N}\mathrm{II}]{} & \multirow[t]{2}{*}{14.53} & ${ }^{3} \mathrm{P}_{1-}{ }^{3} \mathrm{P}_{0}$ & 205.3 & 70.1 & 310 \\
\hline & & ${ }^{3} \mathrm{P}_{2^{-}}{ }^{3} \mathrm{P}_{1}$ & 121.8 & 188 & 48 \\
\hline [Ar II] & 15.76 & ${ }^{2} \mathrm{P}_{1 / 2^{-}}{ }^{2} \mathrm{P}_{3 / 2}$ & 6.99 & 2060 & $3.8 \times 10^{6}$ \\
\hline \multirow[t]{2}{*}[\mathrm{Fe}III]{} & \multirow[t]{2}{*}{16.16} & ${ }^{5} \mathrm{D}_{3}-{ }^{5} \mathrm{D}_{4}$ & 22.93 & 627 & $4.0 \times 10^{4 \mathrm{c}}$ \\
\hline & & ${ }^{5} \mathrm{D}_{2-}{ }^{5} \mathrm{D}_{3}$ & 33.04 & 1063 & $2.1 \times 10^{4 \mathrm{c}}$ \\
\hline [P III] & 19.73 & ${ }^{2} \mathrm{P}_{3 / 2^{-}}{ }^{2} \mathrm{P}_{1 / 2}$ & 17.89 & 804 & $1.1 \times 10^{4 \mathrm{~d}}$ \\
\hline [Ne II] & 21.56 & ${ }^{2} \mathrm{P}_{3 / 2^{-}}{ }^{2} \mathrm{P}_{1 / 2}$ & 12.81 & 1123 & $6.6 \times 10^{5}$ \\
\hline \multirow[t]{2}{*}{ [S III] } & \multirow[t]{2}{*}{23.33} & ${ }^{3} \mathrm{P}_{1-}{ }^{3} \mathrm{P}_{0}$ & 33.46 & 430 & $2.1 \times 10^{3}$ \\
\hline & & ${ }^{3} \mathrm{P}_{2^{-}}{ }^{3} \mathrm{P}_{1}$ & 18.71 & 1199 & $1.7 \times 10^{4}$ \\
\hline \multirow[t]{2}{*}{ [Ar III] } & \multirow[t]{2}{*}{27.63} & ${ }^{3} \mathrm{P}_{1-}{ }^{3} \mathrm{P}_{2}$ & 8.99 & 1600 & $5.4 \times 10^{4}$ \\
\hline & & ${ }^{3} \mathrm{P}_{0^{-}}{ }^{3} \mathrm{P}_{1}$ & 21.84 & 2259 & $2.1 \times 10^{5}$ \\
\hline [N III] & 29.60 & ${ }^{2} \mathrm{P}_{3 / 2^{-}}{ }^{2} \mathrm{P}_{1 / 2}$ & 57.32 & 250 & $2.0 \times 10^{3}$ \\
\hline$[\mathrm{S}$ IV] & 34.83 & ${ }^{2} \mathrm{P}_{3 / 2^{-}}{ }^{2} \mathrm{P}_{1 / 2}$ & 10.51 & 1369 & $2.8 \times 10^{4}$ \\
\hline \multirow[t]{2}{*}{ [O III $]$} & \multirow[t]{2}{*}{35.12} & ${ }^{3} \mathrm{P}_{1-}{ }^{3} \mathrm{P}_{0}$ & 88.36 & 163 & 510 \\
\hline & & ${ }^{3} \mathrm{P}_{2^{-}}{ }^{3} \mathrm{P}_{1}$ & 51.82 & 441 & $3.6 \times 10^{3}$ \\
\hline \multirow[t]{2}{*}[\mathrm{Ne}III]{} & \multirow[t]{2}{*}{40.96} & ${ }^{3} \mathrm{P}_{1-}{ }^{3} \mathrm{P}_{2}$ & 15.55 & 925 & $1.4 \times 10^{5}$ \\
\hline & & ${ }^{3} \mathrm{P}_{0^{-}}{ }^{3} \mathrm{P}_{1}$ & 36.02 & 1325 & $2.0 \times 10^{4}$ \\
\hline
\end{tabular}

${ }^{\text {a }}$ Collision partner is electron and $T=10^{4} \mathrm{~K}$. Calculated from A-coefficient by Shibai (1992) and the collision strength by Osterbrock (1989) except for those marked.

b A-coefficient is from Quinet et al. (1996) and the collision strength is from Zhang \& Pradhan (1995) (IRON project).

c A-coefficient is from Nahar \& Pradhan (1996) and the collision strength is from Zhang (1996) (IRON project).

${ }^{\mathrm{d}}$ A-coefficient is from CLOUDY ver.05.07.06 and the collision strength is from Saraph \& Storey (1999) (IRON project).

[O I] $63 \mu \mathrm{m}$ is not suitable as an input to the models since it is usually optically thick. The [O I] $146 \mu \mathrm{m}$ and the FIR continuum emission are useful to estimate the PDR properties without the uncertainty of the optical depth of the emissions.

\subsection{The Gas-Phase abundance of Si AND Fe}

Then we estimate the gas-phase abundance of $\mathrm{Si}$ and $\mathrm{Fe}$. In the ionized gas, there are a few choices of the reference emission lines to estimate the abundance. Since either of them has advantages and disadvantages, we propose to estimate the gas-phase abundance with all of them and examine the consistency.

The gas-phase Si abundance in the ionized gas is estimated by the [Si II] $35 \mu \mathrm{m}$ to the [N II] $122 \mu \mathrm{m}$ or [S III] $33 \mu \mathrm{m}$. In terms of the ionization potential, $\mathrm{N}^{+}$has an advantage since the ionization potential of $\mathrm{S}^{2+}$ is much higher than that of $\mathrm{Si}^{+}$. In addition, $\mathrm{N}$ is not depleted in the ISM, whereas $\mathrm{S}$ is depleted at some extent in dense regions (Martín-Hernández et al. 2002). On the other hand, [Si II] $35 \mu \mathrm{m} /[\mathrm{S} \mathrm{III}] 33 \mu \mathrm{m}$ is insensitive to the extinction. The elemental abundance ratio of $\mathrm{Si} / \mathrm{S}$ is insensitive to the metallicity because both $\mathrm{Si}$ and $\mathrm{S}$ are $\alpha$-elements. This is a strong advantage when we examine the gas-phase abundance in extragalactic star-forming regions.

The gas-phase Fe abundance in the ionized gas is also estimated by the [Fe II] $26 \mu \mathrm{m} /[\mathrm{N} \mathrm{II}] 122 \mu \mathrm{m}$ and [Fe III] $23 \mu \mathrm{m} /[\mathrm{S} \mathrm{III}] 33 \mu \mathrm{m}$. $\mathrm{Fe}^{2+}$ and $\mathrm{S}^{2+}$ have similar ionization potentials. In addition, both [Fe II] $26 \mu \mathrm{m}$ and [Fe III] $23 \mu \mathrm{m}$ emissions against the Hu $\alpha 12.4 \mu \mathrm{m}$ emission provide the absolute gas-phase abundance after the correction for the ionization fraction as shown in Lebouteiller et al. (2008). Although the solar abundance cannot be assumed as the total elemental abundance even for the first approximation except for nearby regions, the correlation with the metallicity or properties of environments will provide crucial information on the life-cycle of elements.

The gas-phase abundance of $\mathrm{Si}$ and $\mathrm{Fe}$ in the PDR gas are estimated using the PDR models with the physical properties derived in the method described in the previous subsection. According the PDR model by Kaufman et al. (2006), the [Si II] $35 \mu \mathrm{m} /[\mathrm{O}$ I] $146 \mu \mathrm{m}$ is less sensitive to $n$ and $G_{0}$ in $n \sim 10^{2}-10^{4} \mathrm{~cm}^{-3}$ and $G_{0} \sim 1-10^{5}$ (figure 4). Thus observations of [O I] $146 \mu \mathrm{m}$ are essential. 

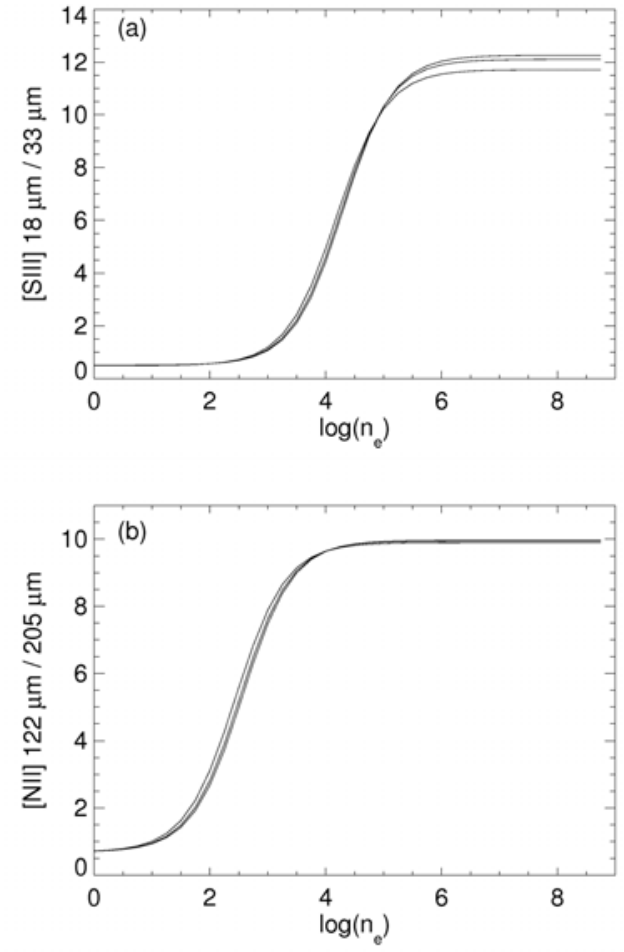

Figure 3. Calculated (a) [S III] $18.7 \mu \mathrm{m} / 33 \mu \mathrm{m}$ and (b) [NII] $122 \mu \mathrm{m} / 205 \mu \mathrm{m}$ against the electron density $\left(n_{e}\right)$. The electron temperature is $7000 \mathrm{~K}, 10000 \mathrm{~K}$, and $12000 \mathrm{~K}$ from lower to upper for (a) and upper to lower for (b).

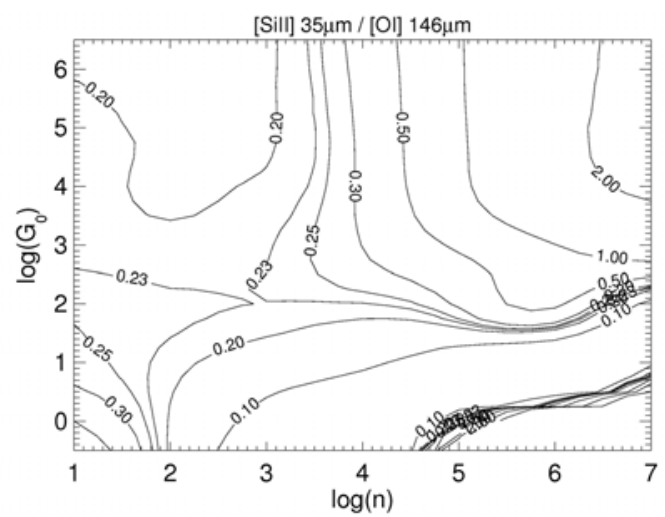

Figure 4. The model estimate of [Si II] $35 \mu \mathrm{m} /[\mathrm{O}$ I] $146 \mu \mathrm{m}$ as a function of $n$ and $G_{0}$ by Kaufman et al. (2006).

\section{SUMmary}

The depletion study of $\mathrm{Si}$ and Fe will provide key information on chemical compositions of dust grains in various environments. In the present paper, we propose the strategy to estimate the gas-phase abundance of $\mathrm{Si}$ and Fe as well as the physical properties using MIR and FIR emission lines that SPICA can observe. To derive the gasphase abundance of $\mathrm{Si}$ and $\mathrm{Fe}$ in both the ionized gas and the PDR gas, mapping observations which provide corre- lations between the two emission lines are crucial. We also suggest that the FIR emission lines such as [N II] $122 \mu \mathrm{m}$ and $205 \mu \mathrm{m}$, and [O I] $146 \mu \mathrm{m}$ are important to derive the physical properties of the gas. Accurate estimate of them will improve the accuracy of the estimate of the gas-phase abundance largely.

\section{REFERENCES}

Clegg, P. E., Ade, P. A. R., Armand, C., et al. 1996, A\&A, 315, L38

Colgan, S. W. J., Haas, M. R., Erickson, E. F., et al. 1993, ApJ, 413, 237

Fuente, A., Martin-Pintado, J., Rodriguez-Fernández, N. J., Cernicharo, J., \& Gerin, M. 2000, A\&A, 354, 1053

Gry, C., Swinyard, B., Harwood, A., et al. 2003, The ISO Handbook, vol.III: LWS - The Long Wavelength Spectrometer, version 2.1

Houck, J. R., Charmandaris, V., Brandl, B. R., et al. 2004, ApJS, 154, 18

Kaufman, M. J., Wolfire, M. G., \& Hollenbach, D. J. 2006, ApJ, 644, 283

Kessler, M. F., Steinz, J. A., Anderegg, M. E., et al. 1996, A\&A, 315, L27

Lebouteiller, V., Bernard-Salas, J., Brandl, B., et al. 2008, ApJ, 680, 398

Martín-Hernández, N. L., Peeters, E., Morisset, C., et al. 2002, A\&A, 381, 606

Mizutani, M., Onaka, T., \& Shibai, H. 2004, A\&A, 423, 579

Nahar S. N., \& Pradhan A. K. 1996, A\&AS, 119, 509

Okada, Y., Onaka, T., Miyata, T., et al. 2008, ApJ, 682, 416

Okada, Y., Onaka, T., Nakagawa, T., et al. 2006, ApJ, 640,383

Okada, Y., Onaka, T., Shibai, H., \& Doi, Y. 2003, A\&A, 412, 199

Osterbrock, D. E. 1989, Astrophysics of Gaseous Nebulae and Active Galactic Nuclei (Mill Valley: University Science Books)

Quinet P., Le Dourneuf M., \& Zeippen C. J. 1996, A\&AS, 120, 361

Rosenthal, D., Bertoldi, F., \& Drapatz, S. 2000, A\&A, 356, 705

Saraph, H. E., \& Storey, P. J. 1999 A\&AS, 134, 369

Savage, B. D., \& Sembach, K. R. 1996, ARAA, 34, 279

Shibai, H. 1992, ISAS Internal Rep. 650 (Tokyo: ISAS)

Timmermann, R., Bertoldi, F., Wright, C. M., et al. 1996, A\&A, 315, L281

van den Ancker, M. E., Tielens, A. G. G. M., \& Wesselius, P. R. 2000, A\&A, 358, 1035

Werner, M. W., Roellig, T. L., Low, F. J., et al. 2004, ApJS, 154, 1

Zhang H. L. 1996, A\&AS, 119, 523

Zhang H. L., \& Pradhan A. K. 1995, A\&A, 293, 953 